\title{
CONVEX AND STARLIKE CRITERIA
}

\author{
HERB SILVERMAN
}

(Received 30 April 1997)

\begin{abstract}
We investigate an expression involving the quotient of the analytic representations of convex and starlike functions. Sufficient conditions are found for functions to be starlike of a positive order and convex.
\end{abstract}

Keywords and phrases. Univalent, starlike, convex.

1991 Mathematics Subject Classification. 30C45.

1. Introduction. Let $S$ denote the class of functions $f$ normalized by $f(0)=f^{\prime}(0)-$ $1=0$ that are analytic and univalent in the unit disk $\Delta=\{z:|z|<1\}$. A function $f$ in $S$ is said to be starlike of order $\alpha, 0 \leq \alpha<1$, and is denoted by $S^{*}(\alpha)$ if $\operatorname{Re}\left\{z f^{\prime}(z) / f(z)\right\}>\alpha, z \in \Delta$, and is said to be convex and is denoted by $K$ if $\operatorname{Re}\left\{1+z f^{\prime \prime}(z) / f^{\prime}(z)\right\}>0, z \in \Delta$. Mocanu [9] studied linear combinations of the representations of convex and starlike functions and defined the class of $\alpha$-convex functions. In [8], it was shown that if

$$
\operatorname{Re}\left[\alpha\left(1+z f^{\prime \prime}(z) / f^{\prime}(z)\right)+(1-\alpha) z f^{\prime}(z) / f(z)\right]>0
$$

for $z \in \Delta$, then $f$ is starlike for $\alpha$ real and convex for $\alpha \geq 1$.

In this note, we investigate the properties of functions defined in terms of the quotient of the analytic representations of convex and starlike functions. In particular, we consider the class $G_{b}$ consisting of normalized functions $f$ defined by

$$
G_{b}=\left\{f:\left|\left(\frac{1+z f^{\prime \prime}(z) / f^{\prime}(z)}{z f^{\prime}(z) / f(z)}\right)-1\right|<b, z \in \Delta\right\} .
$$

We determine sharp values of $b$ for which $G_{b} \subset S^{*}(\alpha), 1 / 2 \leq \alpha<1$, and also find values of $b$ for which $G_{b} \subset K$. It is known $([7,10])$ that $K \subset S^{*}(1 / 2)$. We show that $G_{1} \subset$ $S^{*}(1 / 2)-K$. We also find values of $b$ for which $G_{b}$ is not starlike and not univalent.

We make use of the following lemma obtained by Jack in [4].

LEMmA A. Suppose $\omega$ is analytic for $|z| \leq r, \omega(0)=0$ and $\left|\omega\left(z_{0}\right)\right|=\max _{|z|=r}|\omega(z)|$. Then $z_{0} \omega^{\prime}\left(z_{0}\right)=k \omega\left(z_{0}\right), k \geq 1$.

\section{Main results}

THEOREM 1. If $0<b \leq 1$ and $G_{b}$ is defined by (1.2), then $G_{b} \subset S^{*}(2 /(1+\sqrt{1+8 b}))$. The result is sharp for all $b$.

We prove this theorem in an equivalent form, which we write as 
TheOREM 1a. Set $b=(1-\alpha) / 2 \alpha^{2}, 1 / 2 \leq \alpha<1$. Then $G_{b} \subset S^{*}(\alpha)$, with extremal function $z /(1-z)^{2(1-\alpha)}$.

Proof OF THEOREM 1a. It is well known that if $\omega(z)$ is analytic in $\Delta$ with $\omega(0)=$ 0 , then $\operatorname{Re}\left(\frac{1+(1-2 \alpha) \omega(z)}{1-\omega(z)}\right)>\alpha, z \in \Delta$, if and only if $\omega(z)$ is a Schwarz function, i.e., $|\omega(z)|<1$ for $z \in \Delta$ with $\omega(0)=0$. Set

$$
p(z)=\frac{z f^{\prime}(z)}{f(z)}=\frac{1+(1-2 \alpha) \omega(z)}{1-w(z)}
$$

Then

$$
1+\frac{z f^{\prime \prime}(z)}{f^{\prime}(z)}=p(z)+\frac{z p^{\prime}(z)}{p(z)}
$$

and

$$
\left|\left(\frac{1+z f^{\prime \prime}(z) / f^{\prime}(z)}{z f^{\prime}(z) / f(z)}\right)-1\right|=\left|\frac{z p^{\prime}(z)}{(p(z))^{2}}\right|=\left|\frac{2(1-\alpha) z \omega^{\prime}(z)}{(1+(1-2 \alpha) \omega(z))^{2}}\right| .
$$

If $f \notin S^{*}(\alpha)$, then by Lemma A there is a $z_{0} \in \Delta$ for which $\left|\omega\left(z_{0}\right)\right|=1$ and $z_{0} \omega^{\prime}\left(z_{0}\right) \geq$ $\omega\left(z_{0}\right)$. It then follows from (2.3) that $\left|\frac{z_{0} p^{\prime}\left(z_{0}\right)}{\left(p\left(z_{0}\right)\right)^{2}}\right| \geq \frac{2(1-\alpha)}{(2 \alpha)^{2}}$ which contradicts our hypothesis. This completes the proof.

COROLlaRY 1. $G_{1} \subset S^{*}(1 / 2)$.

Proof. Set $b=1$ in Theorem 1 .

COROLLARY 2. If $\operatorname{Re}\left(\frac{z f^{\prime}(z) / f(z)}{1+z f^{\prime \prime}(z) / f^{\prime}(z)}\right)>1 / 2$ for $z \in \Delta$, then $f \in S^{*}(1 / 2)$.

Proof. This follows from Corollary 1 upon noting that for any complex value $w$, $|w-1|<1 \Longleftrightarrow \operatorname{Re}(1 / w)>1 / 2$.

We next give a partial converse to Corollary 1 .

THEOREM 2. If $f \in S^{*}(1 / 2)$, then $\left|\left(\frac{1+z f^{\prime \prime}(z) / f^{\prime}(z)}{z f^{\prime}(z) / f(z)}\right)-1\right|<1$ for $|z|<(2 \sqrt{3}-3)^{1 / 2}=$ $0.68 \ldots$. The result is sharp.

Proof. Set $\left.p(z)=z f^{\prime}(z) / f\right)(z)=1 /(1-\omega(z))$, where $\omega(z)$ is a Schwarz function. We need to find the largest disk $|z|<R$ for which $\left.\mid z p^{\prime}(z) / p(z)\right)^{2}|=| z \omega^{\prime}(z) \mid<1$. Dieudonné [2] found the region of values for the derivative of Schwarz functions. This led to the sharp bound [3],

$$
\left|\omega^{\prime}(z)\right| \leq\left\{\begin{array}{l}
1, \quad r=|z| \leq \sqrt{2}-1 \\
\frac{\left(1+r^{2}\right)^{2}}{4 r\left(1-r^{2}\right)}, \quad r \geq \sqrt{2}-1 .
\end{array}\right.
$$

Since $\left|z \omega^{\prime}(z)\right| \leq\left(1+r^{2}\right)^{2} / 4\left(1-r^{2}\right)=1$ for $r=(2 \sqrt{3}-3)^{1 / 2}$, the proof is complete. $\square$

3. A counterexample. The extreme points of the closed convex hull of convex functions and functions starlike of order $1 / 2$ are identical. See [1]. Since $G_{1} \subset S^{*}(1 / 2)$, one might, also, expect to have $G_{1} \subset K$. Surprisingly, this is not the case. We now construct a function $f \in G_{1}-K$. 
THEOREM 3. $G_{1} \not \subset K$.

Proof. $G_{1} \subset S^{*}(1 / 2)$. Any of $f \in G_{1}$ satisfies $z f^{\prime}(z) / f(z)=1 /(1-\omega(z))$ for some Schwarz function $\omega(z)$. Setting $\alpha=1 / 2$ in (2.3), we see that $f \in G_{1} \Longleftrightarrow\left|z \omega^{\prime}(z)\right|<1$ for $z \in \Delta$, which means that $z \omega^{\prime}(z)$ must, also, be a Schwarz function. Since $1+$ $z f^{\prime \prime}(z) / f(z)=\left(1+z \omega^{\prime}(z)\right) /(1-\omega(z))$, it suffices to construct a Schwarz function $\Omega(z)=z \omega^{\prime}(z)$ for which

$$
\operatorname{Re}\left\{\frac{1+\Omega(z)}{1-\omega(z)}\right\}<0
$$

at some point $z \in \bar{\Delta}$. Let

$$
A=\left\{z \in \Delta:\left|z-z_{0}\right|<10^{-5}, z_{0}=e^{\pi i / 4}=e^{i \theta_{0}}\right\},
$$

and set

$$
\phi(z)=\left(z_{0}+\bar{z}_{0}\right)\left[\left(1-\bar{z}_{0} z\right)^{1 / N}-1\right],
$$

where $N$ is large enough so that $|\phi(z) / z|<10^{-4}$ for $z \in \Delta-A$ and $|\operatorname{Im} \phi(z)|<10^{-8}$ for $z \in A$. Define $\Omega$ by $\Omega(z)=0.9999(z+\phi(z))$.

We first show that $\Omega(z)$ (and, consequently, $\omega(z)$ ) is a Schwarz function and then show that inequality (3.1) holds when $z=z_{0}$.

If

$$
z \in \Delta-A
$$

then

$$
|\Omega(z)| \leq 0.9999(|z|+|\phi(z)|) \leq 0.9999(1.0001)<1 .
$$

If $z \in A$, set $z=z_{0}-\epsilon e^{i \beta}, 0<\epsilon<10^{-5}$, and note that $-2 \cos \theta_{0} \leq \operatorname{Re} \phi(z) \leq 0$. If $\operatorname{Re}(z+\phi(z)) \geq 0$, then $|z+\operatorname{Re} \phi(z)| \leq|z|<1$. If $\operatorname{Re}(z+\phi(z))<0$, then

$$
|z+\operatorname{Re} \phi(z)| \leq \sqrt{\left(\cos \theta_{0}+\epsilon\right)^{2}+\left(\sin \theta_{0}+\epsilon\right)^{2}}<\sqrt{1+4 \epsilon}<1+2 \epsilon<1.0001 .
$$

Thus, if $z \in A$,

$$
|\Omega(z)| \leq 0.9999|z+\operatorname{Re} \phi(z)|+|\operatorname{Im} \phi(z)|<0.9999(1.0001)+10^{-8}=1 .
$$

Therefore, $\Omega(z)$ is a Schwarz function.

We now show that (3.1) holds at $z=z_{0}$ for this choice of $\Omega(z)$. Since

$$
\left|\frac{\Omega(z)}{z}-1\right|=\left|\omega^{\prime}(z)-1\right|<0.0002 \text { for } z \in \Delta-A,
$$

we may write $\omega(z)=z+\eta(z)$, where $|\eta(z)|<0.0003$ for $z \in A$. Note that

$$
\begin{aligned}
\left(\left|1-\omega\left(z_{0}\right)\right|^{2}\right) \operatorname{Re}\left(\frac{1+\Omega\left(z_{0}\right)}{1-\Omega\left(z_{0}\right)}\right) & =\operatorname{Re}\left\{\left(1-\Omega\left(z_{0}\right)\right)\left(1+\overline{\omega\left(z_{0}\right)}\right)\right\} \\
& =\operatorname{Re}\left\{\left(1-0.9999 \bar{z}_{0}\right)\left(1-\bar{z}_{0}-\overline{\eta\left(z_{0}\right)}\right)\right\} \\
& \leq 1-1.9999 \cos \theta_{0}+0.9999 \cos 2 \theta_{0}+2\left|\eta\left(z_{0}\right)\right| \\
& <1-1.9999 \cos (\pi / 4)+0.0006<0 .
\end{aligned}
$$

Hence, the function $f$ for which $1+z f^{\prime \prime}(z) / f^{\prime}(z)=(1+\Omega(z)) /(1-\omega(z))$ must be in $G_{1}-K$. 
4. Convexity. Since $G_{1} \not \subset K$, we can ask if $G_{b} \subset K$ for some $b<1$. In general, $S^{*}(\alpha) \not \subset K$ even for $\alpha$ arbitrary close to 1 ( $b$ close to 0 ). To see this, we note that $f_{n}(z)=z+a_{n} z^{n}$ is in $S^{*}(\alpha)$ if and only if $\left|a_{n}\right| \leq(1-\alpha) /(n-\alpha)$ and $f_{n}(z) \in K$ if and only if $\left|a_{n}\right| \leq 1 / n^{2}$. Thus, $f(z)=z+(1-\alpha) /(n-\alpha) z^{n} \in S^{*}(\alpha)-K$ for $n>2 /(1-\alpha)$.

We next show that there are values of $b$ for which the functions in $G_{b}$ must be convex.

THEOREM 4. $G_{b} \subset K$ for $b \leq \sqrt{2} / 2$.

Proof. Since $f \in G_{b} \subset G_{1} \subset S^{*}(1 / 2)$, we may write $z f^{\prime}(z) / f(z)=1 /(1-\omega(z))$, where $\omega$ is a Schwarz function. For $f \in G_{b}$, we take $\alpha=1 / 2$ in (2.3) to obtain $\left|z \omega^{\prime}(z)\right|$ $<\sqrt{2} / 2$ and, consequently, $|\omega(z)|<\sqrt{2} / 2, z \in \Delta$. We need to show that

$$
\operatorname{Re}\left\{1+z f^{\prime \prime}(z) / f^{\prime}(z)\right\}=\operatorname{Re}\left\{\frac{\left(1+z \omega^{\prime}(z)\right)}{(1-\omega(z))}\right\}>0 .
$$

Since

$$
\begin{aligned}
\left|\arg \left(\frac{1+z \omega^{\prime}(z)}{1-\omega(z)}\right)\right| & \leq\left|\arg \left(1+z \omega^{\prime}(z)\right)\right|+|\arg (1-\omega(z))| \\
& \leq \frac{\pi}{4}+\frac{\pi}{4}=\frac{\pi}{2}
\end{aligned}
$$

the result follows.

In [6], MacGregor found the radius of convexity for $S^{*}(1 / 2)$ to be $(2 \sqrt{3}-3)^{1 / 2}=$ $0.68 \ldots$ Since $G_{1} \subset S^{*}(1 / 2)$, we know that the radius of convexity is at least this large. The following consequence of Theorem 4 is that functions in $G_{1}$ are convex in the disk $|z|<\sqrt{2} / 2$.

COROLlary. If $f \in G_{b}, \sqrt{2} / 2 \leq b \leq 1$, then $f$ is convex in the disk $|z|<\sqrt{2} / 2 b$.

Proof. If $\left|z \omega^{\prime}(z)\right|<1$ for $z \in \Delta$, then $\left|z \omega^{\prime}(z)\right|<t$ for $|z|<t<1$. If $f \in G_{b}$, then $\left|z \omega^{\prime}(z)\right|<b$ for $z \in \Delta$. Hence, $\left|z \omega^{\prime}(z)\right|<\sqrt{2} / 2$ when $|z|<\sqrt{2} / 2 b$.

5. Examples. Theorem 1 gives a sharp order of starlikeness for $G_{b}$ when $0<b \leq 1$, with $G_{1} \subset S^{*}(1 / 2)$. Our methods do not extend to $b>1$, but we expect the order of starlikeness to decrease from $1 / 2$ to 0 as $b$ increases from 1 to some value $b_{0}$ after which functions in $G_{b}$ need not be starlike. We do not have a sharp result for $b>1$, but our next example shows that the univalent functions in $G_{b}$ are not necessarily starlike for $b \geq 11.66$.

The function $h(z)=z(1-i z)^{i-1}$ is spiral-like [11] and, hence, in $S$ because

$$
\operatorname{Re}\left\{e^{\pi i / 4} \frac{z h^{\prime}(z)}{h(z)}\right\}=\frac{1}{\sqrt{2}}\left(\frac{1-|z|^{2}}{|1-i z|^{2}}\right)>0, \quad z \in \Delta .
$$

Since $z h^{\prime}(z) / h(z)=(1+z) /(1-i z)$, we see that $h$ is not starlike for $|z|<a, \sqrt{2} / 2<$ $a<1$. Thus, $f(z)=f_{a}(z)=h(a z) / a$ is not starlike for $z \in \Delta$. Setting $p(z)=z f^{\prime}(z) /$ $f(z)=(1+a z) /(1-a i z)$, we have

$$
\left|\frac{z p^{\prime}(z)}{(p(z))^{2}}\right|=\left|\frac{(1+i) a z}{(1+a z)^{2}}\right| \leq \frac{\sqrt{2} a}{(1-a)^{2}}<11.66
$$


for $a$ sufficiently close to $\sqrt{2} / 2$. Hence, $f \in G_{b}-S^{*}(0)$ for $b=11.66$.

Finally, we show that the functions in $G_{b}$ need not be univalent. In [5], it is shown for $h(z)=z(1-i z)^{i-1}$ that $g(z)=\int_{0}^{z} h(t) / t d t=(1-i z)^{i}-1$ is not in $S$ because $g\left(z_{0}\right)=g\left(-z_{0}\right)$ for $z_{0}=i\left(e^{2 \pi}-1\right) /\left(e^{2 \pi}+1\right),\left|z_{0}\right|=0.996 \ldots$ We, thus, conclude that for $f(z)=g(c z) / c, c=0.997, f \in G_{b}-S$ for $b$ sufficiently large.

ACKNOWLEDGEMENT. This paper was completed while the author was on a sabbatical leave as a visiting scholar at the University of California at San Diego. I would like to express my deep appreciation to Professor Carl FitzGerald for enlightening discussions, especially for his insight and guidance on the example in Theorem 3.

\section{REFERENCES}

[1] L. Brickman, D. J. Hallenbeck, T. H. MacGregor, and D. R. Wilken, Convex hulls and extreme points of families of starlike and convex mappings, Trans. Amer. Math. Soc. 185 (1974), 413-428. MR 49 3102. Zbl 278.30021.

[2] J. Dieudonné, Recherches sur quelques problèmes relatifs aux polynômes et aux fonctiones bornées d'une variable complexe, Ann. Sci. École Norm. Sup. 48 (1931), 247-358 (French). Zbl 003.11904.

[3] P. L. Duren, Univalent functions, Grundlehren der Mathematischen Wissenschaften [Fundamental Principles of Mathematical Science], vol. 259, Springer-Verlag, New York, 1983. MR 85j:30034. Zbl 514.30001.

[4] I. S. Jack, Functions starlike and convex of order $\alpha$, J. London Math. Soc. 3 (1971), no. 2, 469-474. MR 43\#7611. Zbl 224.30026.

[5] J. Krzyz and Z. Lewandowski, On the integral of univalent functions, Bull. Acad. Polon. Sci. Sér. Sci. Math. Astronom. 11 (1963), 447-448. MR 27\#3791. Zbl 137.05202.

[6] T. H. MacGregor, The radius of convexity for starlike functions of order 1/2, Proc. Amer. Math. Soc. 14 (1963), 71-76. MR 27\#283. Zbl 113.05505.

[7] A. Marx, Unintersuchungen über schlichte Abbildungen, Math. Ann. 107 (1932), 40-67 (German). Zbl 005.10901.

[8] S. S. Miller, P. Mocanu, and M. O. Reade, All $\alpha$-convex functions are univalent and starlike, Proc. Amer. Math. Soc. 37 (1973), 553-554. MR 47 2044. Zbl 258.30012.

[9] P. T. Mocanu, Une propriété de convexité generalisée dans la théorie de la représentation conforme, Mathematica (Cluj) 11 (1969), no. 34, 127-133 (French). MR 42\#7881. Zbl 195.36401.

[10] E. Strohhäcker, Beitrage zur Theorie der schlichten Funktionen, Math. Z. 37 (1933), 356380 (German). Zbl 007.21402.

[11] L. Špačk, Contribution à la theorie des fonctions univalents, Časopis Pěst. Mat. 62 (1932), 12-19 (French). Zbl 006.06403.

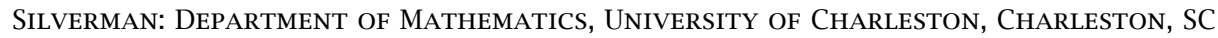
29424, USA 


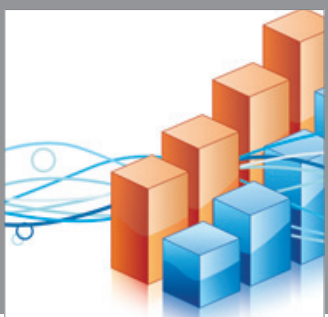

Advances in

Operations Research

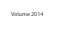

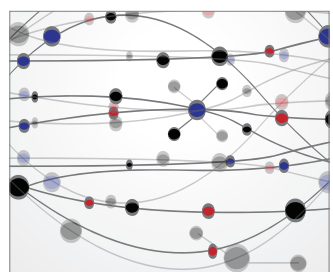

\section{The Scientific} World Journal
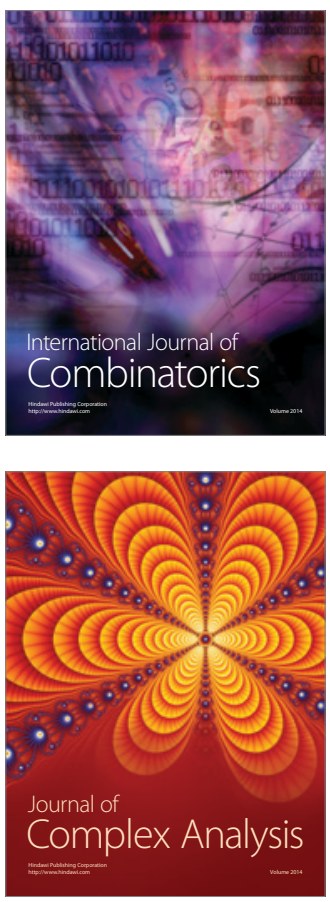

International Journal of

Mathematics and

Mathematical

Sciences
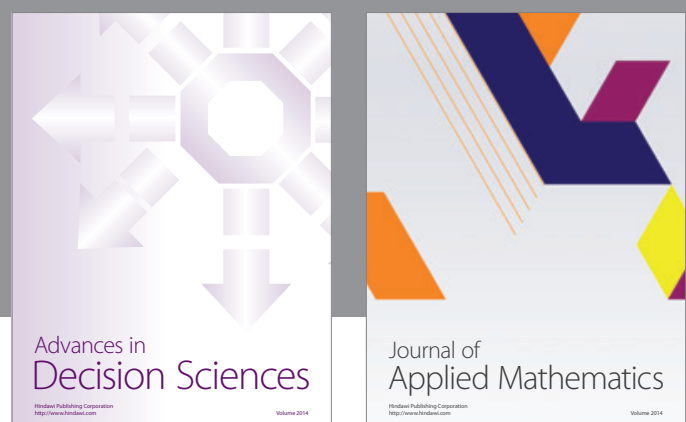

Journal of

Applied Mathematics
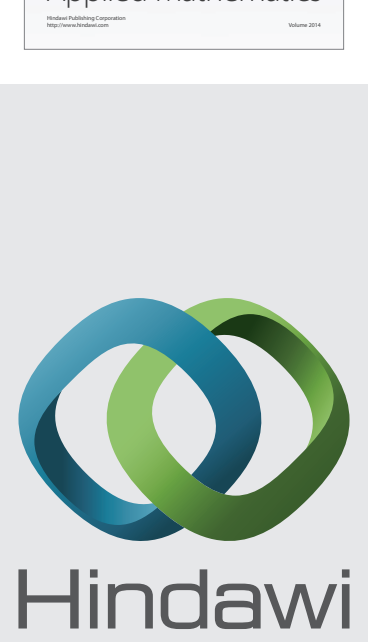

Submit your manuscripts at http://www.hindawi.com
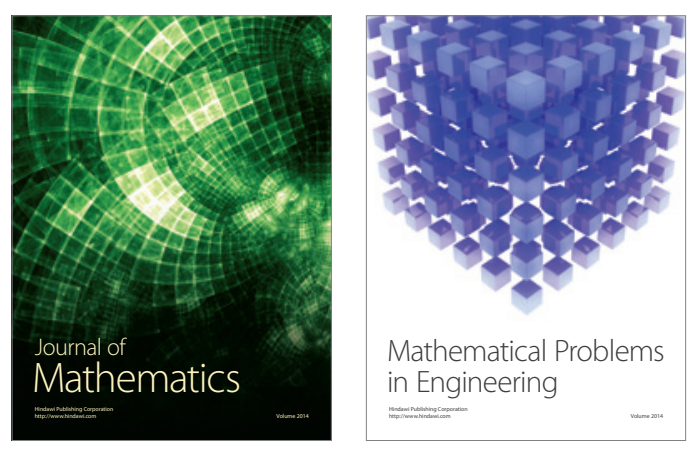

Mathematical Problems in Engineering
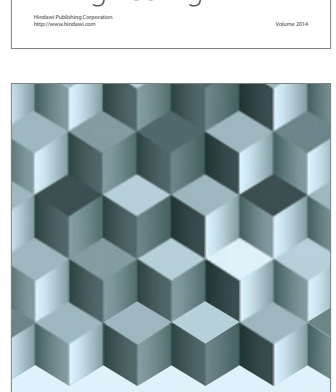

Journal of

Function Spaces
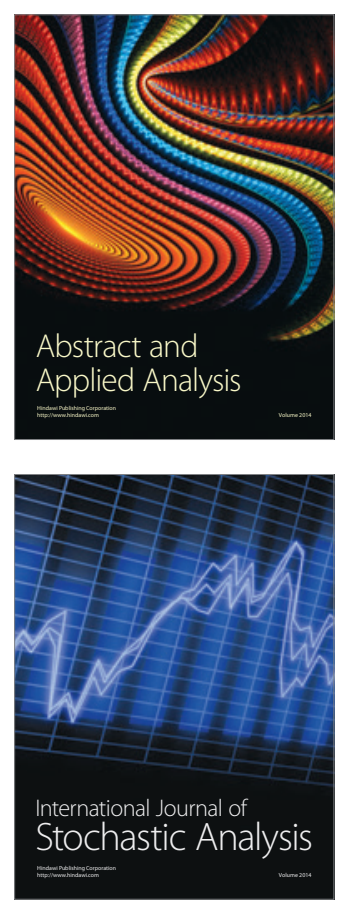

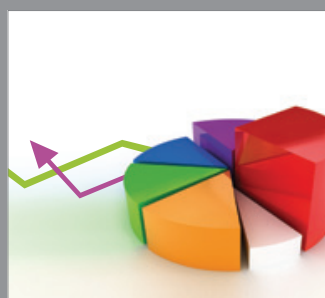

ournal of

Probability and Statistics

Promensencen
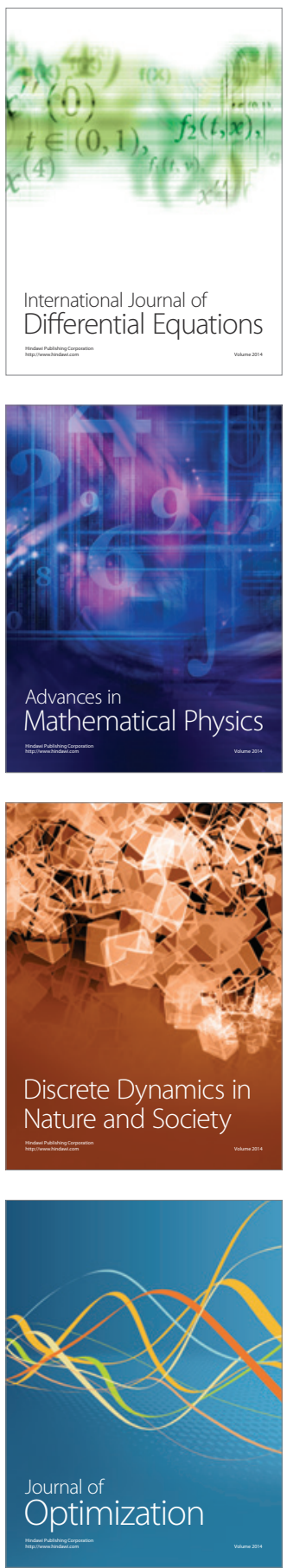